\title{
Editorial: Computational Probability and Mathematical Modeling - A Stochastic Approach in Applied Sciences
}

\author{
José Roberto Cantú-González ${ }^{1 *}$ and F-Javier Almaguer ${ }^{2 *}$ \\ ${ }^{1}$ Escuela de Sistemas PMRV, Campus Acuña, Universidad Autónoma de Coahuila, Saltillo, Mexico, ${ }^{2}$ Facultad de Ciencias \\ Físico Matemáticas, Universidad Autónoma de Nuevo León, San Nicolás de los Garza, Mexico
}

Keywords: mathematical modeling, probability, stochastic processes, simulation, statistics

Editorial on the Research Topic

Computational Probability and Mathematical Modeling - A Stochastic Approach in Applied Sciences

\section{OPEN ACCESS}

Edited and reviewed by:

Daniel Potts,

Technische Universität Chemnitz,

Germany

*Correspondence:

José Roberto Cantú-González

roberto.cantu@uadec.edu.mx

F-Javier Almaguer

francisco.almaguermrt@uanl.edu.mx

Specialty section:

This article was submitted to

Mathematics of Computation and

Data Science,

a section of the journal

Frontiers in Applied Mathematics and

Statistics

Received: 30 July 2019

Accepted: 13 August 2019

Published: 29 August 2019

Citation:

Cantú-González JR and Almaguer F-J

(2019) Editorial: Computational

Probability and Mathematical

Modeling - A Stochastic Approach in

Applied Sciences.

Front. Appl. Math. Stat. 5:45

doi: 10.3389/fams.2019.00045

\section{INTRODUCTION}

Probability and the stochastic processes theory, joint to the mathematical modeling and the respective computational support are clearly important work tools to tackle complex systems, which has been the fundament to develop the Research Topic of "Computational Probability and Mathematical Modeling - A Stochastic Approach in Applied Sciences".

In a brief analysis of the elements contained inside of the Research Topic, we find three important scopes: computational probability, mathematical modeling, and stochastics processes:

Computational probability, it is defined as the development of data structures and algorithms to automate the derivation of existing and new results in probability and statistics [1], Mathematical modeling is the translation of a specific problem from the natural sciences (experimental physics, chemistry, biology, geosciences) or the social sciences, or from technology, into a well-defined mathematical problem [2] and a stochastic process is a probability model that describes the evolution of a system evolving randomly in time [3]. In addition, advances in computer science have been very determinant for growing of science and technology, and all fields of mathematics included stochastic processes have not been bypassed by the digital revolution, because numerical calculation and computer simulation play a decisive role in present-day [4].

Probability and mathematical modeling are usually very adaptable for different proposes, and in definitive, they are the starting point to solve relevant problems [5].

Specifically probability and the stochastic processes theory can be applied in any field where random plays a preponderant role and /or when an analytic treatment is too complicated to be solved. In the other context, mathematical modeling as a descendent from applied mathematics rejects the abstractionism to support the science for understanding of the nature laws; which explains that mathematical models can attend disciplines as physics, biology, finances, economics, any engineering and a variety of fields to solve real problems though its included skills. In this perspective, both probability / stochastic processes theory as the mathematical modeling converge on the same objective: attention of real problems in complex systems. 


\section{RESEARCH TOPIC AND THE ARTICLES CONTAINED}

This Research Topic of "Computational Probability and Mathematical Modeling - A Stochastic Approach in Applied Sciences" presents a little but substantial view of studies related to interesting scenarios from the applied science in fields of genetics, biology, physics, finances, and investment options. In addition, as a core and distinguished common value in these studies, we have that even though all of them come from different disciplines and topics, in a direct or indirect perspective, they possess the essence of probability and mathematical modeling, and they have an objective directed to solve a particular issue. The works contained in this Research Topic combine all the implicit elements of the general theme, and in the most of them it is mentioned the use of simulation as a primary resource in the handling of studied factors.

Individual works in alphabetical order are:

- A Stochastic Phylogenetic Algorithm for Mitochondrial DNA Analysis

- On the Aerodynamic Forces on a Baseball, With Applications

- Output-Feedback Control of Virus Spreading in Complex Networks With Quarantine

- Probabilistic Assessment of Investment Options in Honey Value Chains in Lamu County, Kenya

- Synchronization, Oscillator Death, and Frequency Modulation in a Class of Biologically Inspired Coupled Oscillators

- The Amnesiac Lookback Option: Selectively Monitored Lookback Options and Cryptocurrencies

A Stochastic Phylogenetic Algorithm for Mitochondrial DNA Analysis (Corona-Ruiz et al.):

This research is an exploratory analysis of the mitochondrial DNA and proposes new indices as functions of Shannon entropy, the Chargaff ratio, and fractal dimensions using rescaled-range analysis and DFA; the work suggests to utilize the triplet of indices to construct phylogenetic trees using clustering algorithms. Additionally, it is an initiative to identify the tendencies and correlations in the mutations that produce new species throughout evolutionary history.

On the Aerodynamic Forces on a Baseball, With Applications (Santos et al.):

This article combines experimental results, phenomenological and dimensional analysis; it is classifies as a review, and summarize from recent literature some methods regarding the reproduction and reconstruction of baseball trajectories from aerodynamic forces, and finally discusses their potential applications.

Output-Feedback Control of Virus Spreading in Complex Networks with Quarantine (Alarcn-Ramos et al.):

The research proposes a simple output-feedback control, which stabilizes the extinction state in a virus spreading process over a complex network with quarantine. Additionally, it is provided numerical simulation results to illustrate the functioning of the proposed control scheme for a scale-free network of $N=(10)^{6}$ nodes.
Probabilistic Assessment of Investment Options in Honey Value Chains in Lamu County (Wafula et al.):

This work, classified as method brings an analysis to demonstrate a comprehensive approach to decision-making in a project where decision outcomes are uncertain.

The structure of the decision model includes several strategies, of which the Stochastic Impact Evaluation approach (SIE) and Monte Carlo simulation clearly stands out. SIE approach is used as fundamental base for the decision analysis; particularly SIE prioritizes the critical uncertainties considering the risk factors that may compromise the success and the performance of the project. By the other hand, through the use of Monte Carlo simulation, it was possible to obtain the results of project performance over 10 years, to get it possible the model was ran a total of 10,000 times. This propose is substantially useful for the process of decision-making and the probabilistic assessment of investment options, the case of honey value chains in Lamu County, Kenya is analyzed as study case.

Synchronization, Oscillator Death, and Frequency Modulation in a Class of Biologically Inspired Coupled Oscillators (Franci et al.):

This research is focused on the basic mathematical principles that underlie the emergence of synchronous biological rhythms, in particular, the circadian clock. The study analyzes the role that the coupling strength, coupling type, and noise play in the synchronization of a system of coupled, non-linear oscillators. Authors report the following two phenomena found, whose are described as new from a non-linear collective phenomena perspective: In diffusively coupled cells, resting node dynamics imply global asymptotic stability; oscillating node dynamics imply global-synchronization for small coupling, and multistability between oscillator death and global synchronization for large coupling. In stochastic, linearly coupled populations, it is described the dynamical mechanisms through which coupling modulates the frequency of the synchronous oscillation. Finally, the article and its presented results, emphasizes the importance of simple mathematical models in understanding situations where synchronization of multiple oscillating populations appears. Additionally, Authors believe that results obtained may help to shed light on physiological and pathological phenomena involving synchronization of oscillators in important tissues as Parkinson's disease $[6,7]$, and epilepsy [8, 9].

The Amnesiac Lookback Option: Selectively Monitored Lookback Options and Cryptocurrencies (Chang and Li):

This research suggests the use of its properties to reduce risk exposure in cryptocurrency markets through blockchain enforced smart contracts and correct for informational inefficiencies surrounding prices and volatility. In addition, this work generalizes partial, discretely monitored lookback options that dilute premiums by selecting a subset of specified periods to determine payoff, which authors call amnesiac lookback options. As part of the utilized method, authors price Amnesiac lookbacks with Monte Carlo simulations of Gaussian random walks under equidistant and random periods. The paper concludes that the instrument provides an ideal space for investors to balance their risk, and as a prime candidate to hedge extreme volatility. 


\section{CONCLUDING REMARKS}

This collection of articles included in this volume has provided an interesting view of applications associated to fields of probability, statistics, and mathematic modeling, all of them supported by a computational context; additionally it has been determinant the approach of stochasticity and simulation used in most of them as a key element in the utilized methodology. Each article contained, describes a particular object of study, bring a state of art to support the nature of the research, presents a methodology to solve the defined problem and finally provides results and conclusions as the evidence of the efficiency of the entire propose.

We hope the present collection of articles can be attractive for readers from the academic community of mathematics and the applied sciences in general; also, it is our desire that these papers can provide a starting

\section{REFERENCES}

1. Drew JH, Evans DL, Glen AG, Leemis LM. Computational Probability, Algorithms and Applications in the Mathematical Sciences. Cham: Springer International Publishing (2017).

2. Eck C, Garcke H, Knabner P. Mathematical Modeling. Cham: Springer International Publishing (2017).

3. Kulkarni VG. Introduction to Modeling and Analysis of Stochastic Systems. New York, NY: Springer Science+Business Media, LLC (2011).

4. Schuster P. Stochasticity in Processes, Fundamentals and Applications to Chemistry and Biology. Cham: Springer International Publishing AG (2016).

5. Heinz S. Mathematical Modeling. Berlin; Heidelberg: Springer-Verlag (2011).

6. Hammond C, Bergman H, Brown P. Pathological synchronization in Parkinson's disease: networks, models and treatments. Trends Neurosci. (2007) 30:357-64. doi: 10.1016/j.tins.2007.05.004

7. Schnitzler A, Gross J. Normal and pathological oscillatory communication in the brain. Nat Revi Neurosci. (2005) 6:285-96. doi: 10.1038/nrn1650 point for future researches in solving of practical and complex problems.

\section{AUTHOR CONTRIBUTIONS}

All authors listed have made a substantial, direct and intellectual contribution to the work, and approved it for publication.

\section{ACKNOWLEDGMENTS}

We want to express our recognition to the Universidad Autónoma de Coahuila and the Universidad Autónoma de Nuevo León for providing all the logistical support to ensure the success of this collaborative work. Likewise, we also express our sincere appreciation to Frontiers and all its team for their kind cooperation and their continuous organizational support, their contribution was key to make possible this project.

8. Engel JJ, Bragin A, Staba R, Mody I. High-frequency oscillations: what is normal and what is not? Epilepsy. (2009) 50:598-604. doi: 10.1111/j.1528-1167.2008.01917.x

9. Velazquez J, Carlen P. Gap junctions, synchrony and seizures. Trends Neurosci. (2000) 23:68-74. doi: 10.1016/S0166-2236(99)

Conflict of Interest Statement: The authors declare that the research was conducted in the absence of any commercial or financial relationships that could be construed as a potential conflict of interest.

Copyright (C) 2019 Cantú-González and Almaguer. This is an open-access article distributed under the terms of the Creative Commons Attribution License (CC BY). The use, distribution or reproduction in other forums is permitted, provided the original author(s) and the copyright owner(s) are credited and that the original publication in this journal is cited, in accordance with accepted academic practice. No use, distribution or reproduction is permitted which does not comply with these terms. 